Bio - grafía. Escritos sobre la Biología y su Enseñanza. ISSN 2027-1034

Edición Extraordinaria. p.p. 185 - 193

Memorias del IX Encuentro Nacional de Experiencias en Enseñanza de la Biología y la Educación Ambiental. IV Congreso Nacional de Investigación en Enseñanza de la Biología.

\title{
LA MODELIZACIÓN DE LAS FUNCIONES VITALES EN QUINTO GRADO DE PRIMARIA
}

\author{
MODELLING 'VITAL FUNCTIONS' IN FIFTH GRADE
}

\author{
Aura Yuseli Mena Gómez \\ I.E. Santo Cristo de Zaragoza - Antioquia \\ Fanny Angulo Delgado ${ }^{1}$
}

\section{RESUMEN}

Se propone una forma novedosa para la enseñanza y aprendizaje de las funciones vitales en la escuela, a través del proceso de modelización de la lombriz de tierra como ser vivo, en donde los escolares aprenden a dar sentido a situaciones cotidianas, utilizando un lenguaje científico escolar. El proceso se lleva a cabo teniendo en cuenta el Modelo ONEPSI (Gutiérrez, 2014) para configurar el Modelo Estudiantil inicial (MEi), el Modelo Disciplinar (MD); el Modelo Curricular (MCU); las demandas de aprendizajes de los estudiantes (Leach y Scott, 2002) y el Modelo Científico Escolar de Arribo (MCEA) como meta para alcanzar los objetivos en tanto se constituye en herramienta para el diseño de la secuencia didáctica donde se atienden las demandas. Como resultado, los estudiantes construyen el Modelo Científico Escolar Logrado (MCEL).

PALABRAS CLAVES: Modelización, ser vivo, funciones vitales, secuencia didáctica, demandas de aprendizaje.

\begin{abstract}
This is a new experience for science teaching and learning modeling earthworm vital functions in the school. Students learn to make sense of everyday situations using a scientific school language, That process is carried out taking into account the ONEPSI model (Gutiérrez, 2014) making explicit the initial Student Model (MEi), Scientific Model, Curricular Model, students' learning demands (Leach and Scott, 2002) and the Scientific Model School of Arrival (MCEA) as a goal to achieve the objectives because is a design tool for teaching and learning sequence where student' learning demands are met. As a result, students get a Scientific School Model (MCEL).
\end{abstract}

KEYWORDS: Modeling, living being, teaching and learning sequence, vital functions, learning demands.

\footnotetext{
${ }^{1}$ Universidad de Antioquia
} 
Bio - grafía. Escritos sobre la Biología y su Enseñanza. ISSN 2027-1034

Edición Extraordinaria. p.p. 185 - 193

Memorias del IX Encuentro Nacional de Experiencias en Enseñanza de la Biología y la

Educación Ambiental. IV Congreso Nacional de Investigación en Enseñanza de la Biología.

\section{INTRODUCCIÓN}

Enseñar ciencia no es tarea fácil para el docente, generalmente sus contenidos son abordados de forma fragmentada lo cual obstaculiza el proceso de construcción de la relación causa- efecto y que el estudiante pueda explicar dando sentido a los fenómenos que ocurren en su entorno, como es el caso de las funciones vitales. Desde este punto de vista, una de las tareas desde la enseñanza de la ciencia es que los escolares puedan construir modelos, los cuales, permitan entender, explicar y predecir los fenómenos que ocurren dentro de su cuerpo en su diario vivir.

Por lo anterior, en este artículo se presenta un proceso de modelización en la enseñanza de las ciencias naturales, donde se usan las demandas de aprendizaje encontradas en el Modelo Estudiantil inicial, para la construcción de la secuencia didáctica y el Modelo ONEPSI, como una herramienta para la organización del lenguaje social cotidiano de los escolares y el análisis de sus modelos. Luego, se presentan los resultados hallados en las explicaciones de los niños.

Según García (2005), aprender ciencias naturales debe servir para que los escolares expliquen hechos del mundo, por ejemplo: porque el cuerpo de una lombriz de tierra es capaz de autorepararse cuando sufre un daño, porque el cuerpo humano suda, elimina líquidos en la orina, o porque los hijos se parecen a sus progenitores. Para ello, es necesario que los niños tengan la capacidad de elaborar modelos cada vez más complejos que les permitan establecer relaciones.

\section{OBJETIVO}

Presentar el proceso de modelización y su evolución acerca de las funciones vitales como fenómeno educativo de valor, usando el Modelo ONEPSI y las demandas de aprendizajes encontradas en el Modelo Estudiantil inicial, para la construcción de la secuencia de enseñanza y aprendizaje.

\section{REFERENTES CONCEPTUALES}

El referente de la investigación es la "Modelización en la enseñanza de las ciencias". Este enfoque epistemológico permite caracterizar las actividades científicas escolares y elaborar materiales didácticos fundamentados teóricamente. En esta investigación lo primero que se hizo fue seleccionar el fenómeno a modelizar con valor educativo para los estudiantes, en este caso, se eligió el ser vivo "lombriz de tierra" para estudiar las funciones vitales; este animal se tomó como objeto de estudio porque su manipulación no genera ningún peligro para los estudiantes, además de eso, es fácil de conseguir y de elaborar las condiciones ambientales artificiales para su posterior estudio. Luego se construyeron las primeras actividades de la secuencia de enseñanza y aprendizaje, con el fin de conocer los Modelos Estudiantiles iniciales (MEi) de los niños; la información recolectada, se organizó en una tabla que contiene los componentes ontológicos, epistemológicos y psicológicos, dentro de los cuales se encuentran los constituyentes del 


\title{
Bio - grafía. Escritos sobre la Biología y su Enseñanza. ISSN 2027-1034
}

Edición Extraordinaria. p.p. 185 - 193

\author{
Memorias del IX Encuentro Nacional de Experiencias en Enseñanza de la Biología y la \\ Educación Ambiental. IV Congreso Nacional de Investigación en Enseñanza de la \\ Biología.
}

modelo (entidades, propiedades, relaciones e inferencias). La actividad de comunicación de objetivos que consistió en conversar, salir al campo con los estudiantes y por último construir una historieta, permitió conocer los MEi.

Después se construyó una tabla que contiene el modelo curricular $(\mathrm{MCu})$ con los aportes que se encuentran de manera implícita en el plan de estudio de la institución educativa; pero se tiene presente el propósito del aprendizaje esperado del ser vivo, en este modelo se identifica el conocimiento que se imparte desde el entorno escolar. Se continuó con la elaboración de una tabla con el contenido del modelo disciplinar (MD), el cual consiste en un modelo adaptado por las representaciones de la ciencia, donde se organizó un conjunto de entidades con sus principales propiedades y los enunciados legales que determinan el comportamiento de las entidades del ser vivo. Las funciones esenciales de este modelo son las explicaciones y predicciones desde el punto de vista de la ciencia (Gutiérrez, 2014, p.51).

Este modelo científico fue utilizado como una herramienta para presentar al ser vivo y las funciones vitales, por lo tanto, se convierte para el docente en un elemento útil a la hora de enseñar las funciones vitales, debido a que el estudiante construye desde este modelo una representación del fenómeno que lo lleva a obtener una experiencia del mundo real, de esta manera sistematiza, simplifica y reestructura los conceptos que tiene de la ciencia (Galagovsky y Adúriz-Bravo, 2001).

El MCEA surge al comparar tres modelos (MEi, MCu y MD) en donde se identifican las similitudes, diferencias y relaciones entre sus constituyentes (entidades, propiedades, relaciones y las inferencias). Lo que se pretende con el MCEA, es acercar a los estudiantes al contenido de la ciencia escolar; donde se expresa lo que se espera que los estudiantes aprendan mediante la implementación de la secuencia de enseñanza y aprendizaje, dirigida a la modelización del fenómeno educativo a partir de la "lombriz de tierra" como ser vivo. También sirvió para el diseño y validación de la secuencia didáctica (López \& Moreno, 2014, p.11).

Por último, se identificaron las demandas de aprendizaje de los estudiantes tensionando el MCEA y el MEi a partir de sus componentes ontológicos, epistemológicos y psicológicos, en una tabla donde se incorporaron diferentes puntos de vista como son: el lenguaje social cotidiano de los estudiantes, el lenguaje de la ciencia escolar, el lenguaje de la ciencia, las demandas de aprendizaje y el tipo de demanda, con el fin de identificar las necesidades de los escolares y asimismo diseñar las actividades de la secuencia de enseñanza y aprendizaje dirigida a modelizar el fenómeno (Leach y Scott, 2002).

Para comprender las representaciones de los estudiantes y su evolución, se diseñó una secuencia que busca observar el proceso de modelización de las funciones vitales a lo largo de su aplicación, como se muestra en la imagen: 
Bio - grafía. Escritos sobre la Biología y su Enseñanza. ISSN 2027-1034

Edición Extraordinaria. p.p. 185 - 193

Memorias del IX Encuentro Nacional de Experiencias en Enseñanza de la Biología y la Educación Ambiental. IV Congreso Nacional de Investigación en Enseñanza de la Biología.

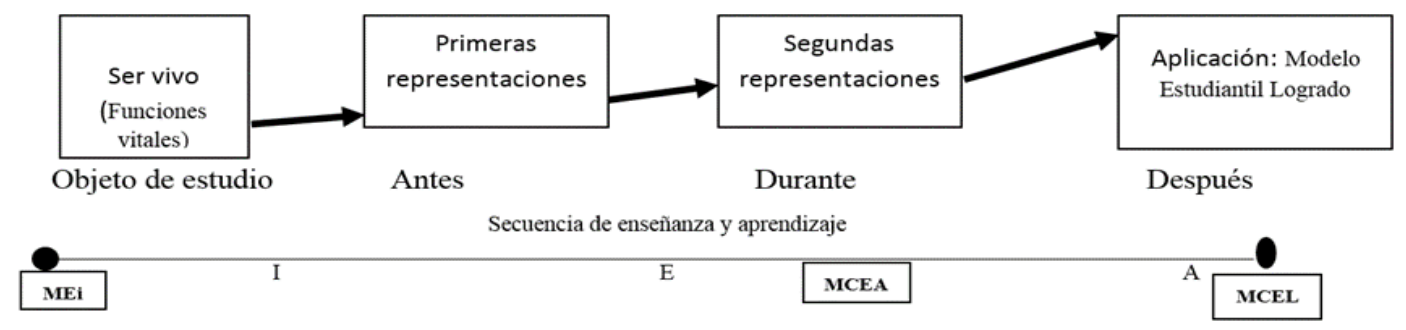

Figura 1. Descripción de proceso de modelización durante las etapas de investigación.

\section{METODOLOGÍA}

La investigación se desarrolló en el departamento de Antioquia (Colombia), en la subregión del Bajo Cauca en el municipio de Zaragoza, en la Institución Educativa Santo Cristo de Zaragoza, teniendo como muestra 45 estudiantes del grado $5^{\circ}$ de primaria, cuyas edades oscilan entre 9 y 11 años. La metodología que fue utilizada en este proyecto de investigación está enmarcada bajo el paradigma cualitativo con un estudio de caso único longitudinal (Rodríguez, Gil \& García, 1996).

Para analizar la información recolectada producto del trabajo realizado con todos los estudiantes, en la fase uno, se construyó una red sistémica que sirvió como insumo en el análisis y ordenamiento de los datos obtenidos en la tabla de MEi; en la fase dos y tres se utilizó el programa NVIVO para la organización, clasificación y procesamiento de datos, en donde los registros se archivaron de forma separada de acuerdo con las secuencias de las actividades, de esta manera se observó la evolución de los modelos y explicaciones, para ello, fue necesario elaborar bitácoras de análisis y la categorización de los datos, con el fin de obtener información clara y precisa. Cada bloque se analizó teniendo encuentra el Modelo ONEPSI, las diferencias y semejanzas en las explicaciones de los niños.

\section{RESULTADOS}

El análisis de los datos se realizó en función de las fases propuestas en la secuencia de enseñanza, utilizando el Modelo ONEPSI para la interpretación de los Modelos Científicos Escolares Logrados (MCEL). Lo anterior sirvió como criterio de evaluación de los modelos construidos.

\section{Los modelos iniciales acerca del ser vivo}

Para explicitar el MEi se pidió a los escolares realizar una historieta acerca de un día de vida de la lombriz de tierra. Este fue uno de los resultados obtenidos en la fase 1 de la secuencia. 
Bio - grafía. Escritos sobre la Biología y su Enseñanza. ISSN 2027-1034

Edición Extraordinaria. p.p. 185 - 193

Memorias del IX Encuentro Nacional de Experiencias en Enseñanza de la Biología y la Educación Ambiental. IV Congreso Nacional de Investigación en Enseñanza de la Biología.

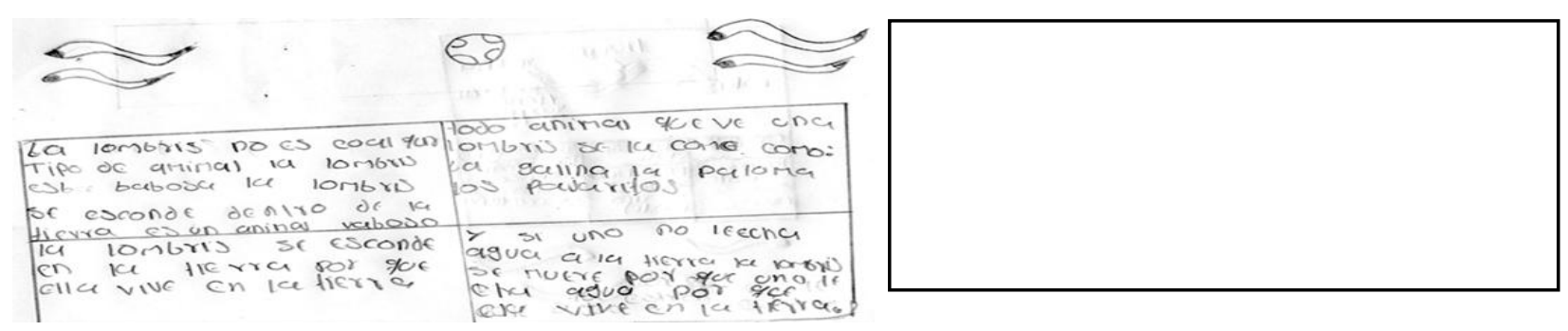

Figura 1: Actividad de la historieta del estudiante $n^{\circ} 8$. Fase 1 Continuación de la exploración del MEi.

En este MEi se observa que el estudiante no utiliza los conceptos de las funciones vitales y la palabra célula para dar explicaciones sobre un hecho del mundo, por este motivo las demandas son ontológicas, epistemológicas y psicológicas de acuerdo con el Modelo ONEPSI, porque presenta dificultades conceptuales para dar explicación a los fenómenos, ya que piensa que el animal es un ser vivo que tiene características diferentes en comparación con otros animales terrestres, como es el tener en su cuerpo baba y no por presentar las funciones de nutrición, relación y reproducción, además en su modelo anatómico (observar el dibujo de la lombriz de tierra arriba de las explicaciones) no utiliza estructuras anatómicas para ayudarse en las explicaciones.

Al dibujar, los escolares representan sus imágenes e ideas, las cuales se convierten en una herramienta para expresar la compresión de la realidad percibida.

El diálogo y las expresiones gráficas facilitaron la construcción del MEi, teniendo en cuenta los constituyentes del modelo ONEPSI.

Tabla 1

Modelo Estudiantil inicial - MEi sobre el fenómeno educativo de valor: la lombriz de tierra como ser vivo

Constituyentes
ontológicos

\section{Constituyentes Epistemológicos}

Constituyentes Psicológicos

Enunciados Legales

$\begin{array}{lll}\begin{array}{c}\text { Entida } \\ \text { des }\end{array} & \text { Propiedades } & \begin{array}{c}\text { Relacio Inferencias } \\ \text { nes }\end{array}\end{array}$

ar
(Causalidad)
Observ
Predecir
Inferencia
Explicar 
Bio - grafía. Escritos sobre la Biología y su Enseñanza. ISSN 2027-1034

Edición Extraordinaria. p.p. 185 - 193

Memorias del IX Encuentro Nacional de Experiencias en Enseñanza de la Biología y la

Educación Ambiental. IV Congreso Nacional de Investigación en Enseñanza de la Biología.

Ser vivo

\begin{tabular}{ll}
$\begin{array}{r}\text { Ser vivo } \\
\text { invertebrado }\end{array}$ & \multicolumn{1}{c}{-La lombriz baba, cola, } \\
que nace, & rayitas negras \\
respira, se & en la barriga \\
reproduce, & estómago y \\
crece se & habla con otras \\
alimenta & lombrices, es de \\
tiene hijos y & color rosado y \\
muere. & blanco, tiene 5 \\
& corazones, \\
& bolas, ojos, \\
& intestino delgado \\
& y tripa. Es \\
& pálida, flaca, \\
& como enferma.
\end{tabular}

- Es larga se estira mucho, es gorda. Por dentro no tiene huesos.

-A la lombriz se la come el pollito y la gallina.
-La

lombriz no

puede vivir

sin agua

porque se

seca.

-La

lombriz si se parte en dos sigue viva.

-Cuando no hay agua la lombriz se muere $y$ cuando hay agua no se muere.

\section{-Si la} lombriz no existiera las plantas y los árboles tampoco existieran.
- No camina con los pies sino que se estira como una culebra. Además no puede caminar muy rápido porque la estructura que tiene no la deja.

-La lombriz cuando se parte por la mitad tiene que salir de la tierra para poder hidratarse.

La lombriz tiene baba para que no se seque. Hace un hueco hondo para que no se la coman los humanos.
Los niños se imaginan que la lombriz es un ser vivo porque se puede reproducir como los humanos; además describen a la lombriz como un animal invertebrado porque no tiene huesos en su estructura pero se imaginan que por dentro del animal hay tripas e intestinos que reparten los alimentos.

También argumentan que este ser vivo se arrastra para buscar su alimento y de esta manera logra sobrevivir.

En la fase 2 se aplicó la secuencia de enseñanza dirigida al proceso de modelización. Se presenta un fragmento con su respectivo ciclo.

\begin{tabular}{|c|c|c|c|}
\hline $\begin{array}{l}\text { b Fase 2: de evolución } \\
\text { de los modelos y } \\
\text { regulación de los } \\
\text { aprendizaje. }\end{array}$ & $\begin{array}{l}\text { 4. SEA } \\
\text { Parte D }\end{array}$ & $\begin{array}{l}\text { b.4.1 Act anticipación de los contenidos } \\
\text { b.4.2 Act de introducción } \\
\text { b.4.3 Act de estructuración } \\
\text { b.4.4 Act de aplicación }\end{array}$ & $\begin{array}{l}\text { b.4.1.1 Identificación de la lombriz como ser vivo. } \\
\text { b.4.2.1 La lombriz y el ambiente "función de } \\
\text { relación". } \\
\text { b. } 4.3 .1 \text { Práctica de campo } \\
\text { b.4.4.1 Identificación de la lombriz de tierra como } \\
\text { ser vivo. }\end{array}$ \\
\hline
\end{tabular}

Figura 2. Secuencia de enseñanza y aprendizaje parte 4. 
Bio - grafía. Escritos sobre la Biología y su Enseñanza. ISSN 2027-1034

Edición Extraordinaria. p.p. 185 - 193

Memorias del IX Encuentro Nacional de Experiencias en Enseñanza de la Biología y la

Educación Ambiental. IV Congreso Nacional de Investigación en Enseñanza de la Biología.

Con las actividades b.4.1.1 y b.4.2.1 se pudo notar un cambio significativo en las explicaciones de los escolares, donde incorporaron entidades y propiedades cuando se realiza una pregunta problema basada en los movimientos de la lombriz.

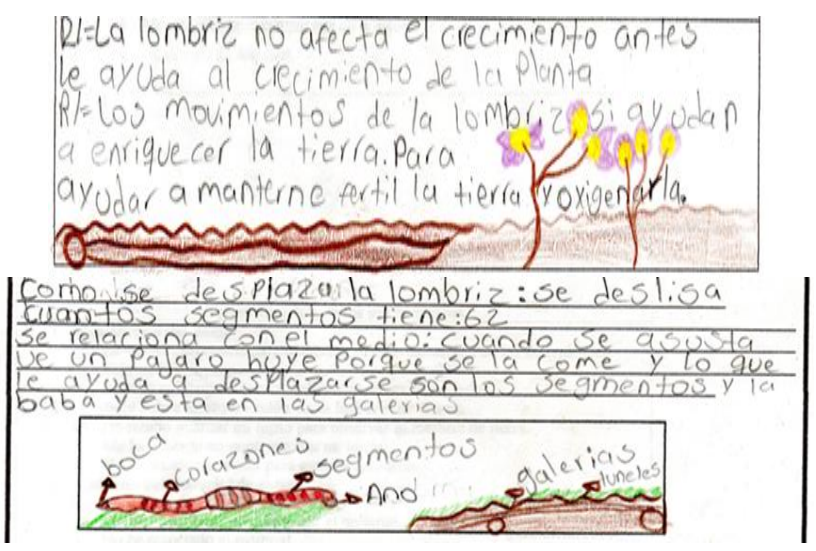

Figura 3. Representación del hábitat y movimiento de la lombriz debajo de la tierra.

En este caso, el escolar representa lo que pasa con la lombriz y la planta a partir de una imagen. Se puede notar cómo los estudiantes empiezan a comprender el papel que cumple la lombriz en la tierra, surge un modelo anatómico incipiente del animal, donde incorporan entidades como los segmentos y las galerías; pero no solo eso, reconocen que su estructura ayuda al desplazamiento y huir frente al peligro. Estas pequeñas explicaciones permiten observar cómo los estudiantes usan herramientas conceptuales para establecer relaciones. En la actividad de aplicación se pregunta a los escolares que pasaría si la lombriz sufre una lesión, la respuesta de los escolares fue la siguiente:

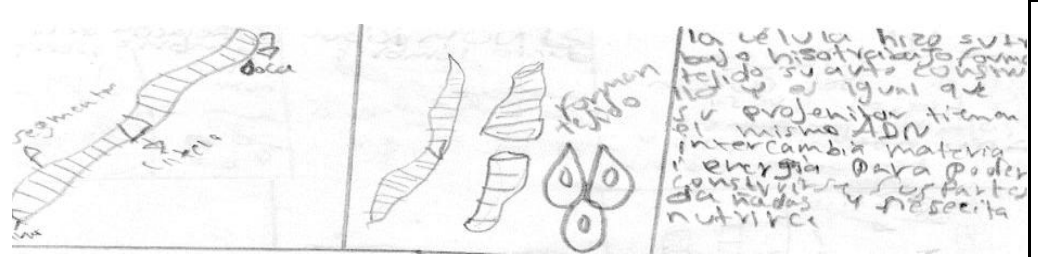

Figura 4. Explicación de los escolares de la reconstrucción de la lombriz como ser vivo.
Segmento, clitelo, boca y ano. Forman tejido.

La célula hiso su trabajo su autoconstruyo y es igual que su progenitor, tienen el mismo $A D N$, intercambian materia $y$ energía para poder construirse sus partes dañadas y necesita nutrirse.

En este caso se observan nociones ontológicas, epistemológicas y psicológicas, en las explicaciones de los estudiantes, puesto que pueden ser capaces de expresarlo; cuando un estudiante no es capaz de representar sus ideas, el proceso de modelización 
Bio - grafía. Escritos sobre la Biología y su Enseñanza. ISSN 2027-1034

Edición Extraordinaria. p.p. 185 - 193

Memorias del IX Encuentro Nacional de Experiencias en Enseñanza de la Biología y la

Educación Ambiental. IV Congreso Nacional de Investigación en Enseñanza de la Biología.

no se está llevando a cabo en el sujeto que aprende, del mismo modo, si no comunica lo aprendido difícilmente podrán interpretar y construir nuevos conocimientos.

Fase tres. Proceso de modelización

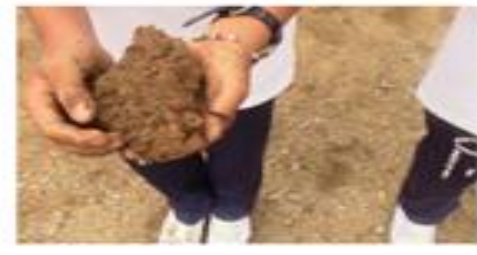

Objeto real
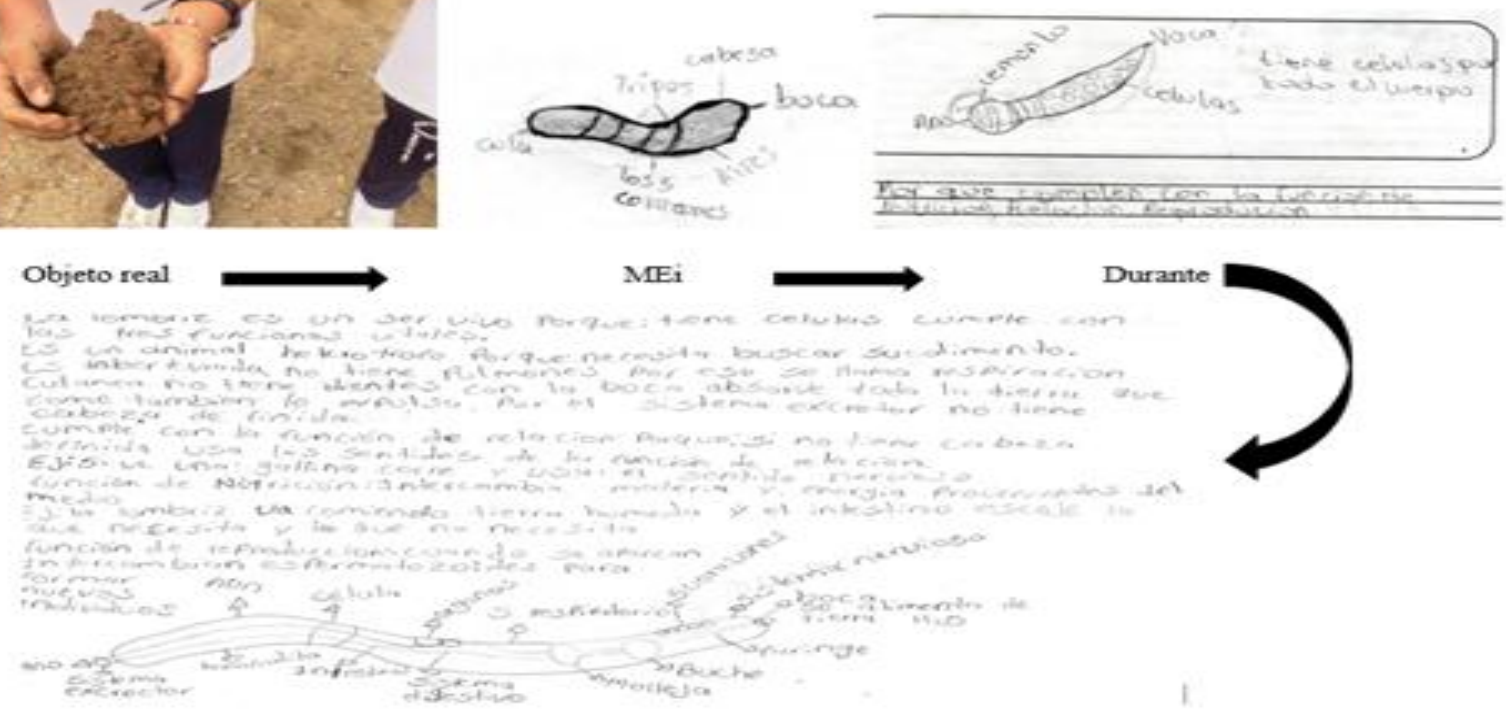

MCEL

Figura 5. Actividad de intercambio de materia y energía.

La lombriz es un ser vivo por que tiene células y cumple con las tres funciones vitales, es un animal heterótrofo, porque necesita buscar su alimento. Es invertebrada, no tienen pulmones por eso se llama respiración cutánea, no tienen diente con la boca absorbe toda la tierra que come también lo expulsa por el sistema excretor, no tienen cabeza definida. Cumple con la función de relación porque: si no tienen cabeza definida usa los sentidos de la función de relación. Ejemplo si ve una gallina corre y usa el sentido nervioso. Función de nutrición: intercambia materia y energía proveniente del medio. Ejemplo: la lombriz va comiendo tierra humedad y el intestino escoge lo que necesita y lo que no necesita. Función de reproducción: cuando se aparean intercambian espermatozoides para formar nuevos individuos. Dibujo de la lombriz: ADN, célula, órganos, s. respiratorio, 5 corazones, sistema nervioso, boca, se alimenta de tierra, faringe, buche, molleja, sistema digestivo, intestinos, hermafrodita, sistema excretor y ano.

\section{Transcripción de la actividad.}

Este es el MCEL, donde los estudiantes presentan un modelo anatómico. Para ellos la lombriz de tierra es un ser vivo porque tiene células y cumple con las funciones vitales, las cuales lo ayudan a mantenerse con vida, además argumentan que cada una de las funciones son necesarias para el desempeño de las actividades del animal.

\section{CONCLUSIÓN}


Bio - grafía. Escritos sobre la Biología y su Enseñanza. ISSN 2027-1034

Edición Extraordinaria. p.p. 185 - 193

Memorias del IX Encuentro Nacional de Experiencias en Enseñanza de la Biología y la Educación Ambiental. IV Congreso Nacional de Investigación en Enseñanza de la Biología.

Con base en los resultados, el proceso de modelización en la clase de ciencias es un método para acercar a los estudiantes a un lenguaje científico escolar, ya que se observa una evolución en las explicaciones de los niños en cuanto a los Modelos Estudiantiles iniciales, justifican sus respuestas utilizando modelos más complejos para explicar fenómenos. La secuencia de enseñanza y aprendizaje permitió que ontológicamente los estudiantes incorporaran nuevas entidades al referirse al fenómeno de las funciones vitales, como se observa en el MCEL.

El modelo que presentó mayor evolución fue el de la reproducción, pues se observa que los niños lograron incorporar algunas entidades que se encontraban ausentes en sus modelos explicativos y realizaron representaciones coherentes con los conceptos trabajados.

\section{BIBLIOGRAFÍA}

Galagovsky, L. \&. Adúriz-Bravo (2001). Modelos y analogías en la enseñanza de las ciencias naturales. El concepto de modelo didáctico analógico. Enseñanza de las Ciencias, 19(2) ,31-242.

García, M.P. (2005) Los modelos como organizadores del currículo en biología. Enseñanza de las Ciencias. Número Extra.1-6.

Gutiérrez, R. (2014). Lo que los profesores de ciencia conocen y necesitan conocer acerca de los modelos: aproximaciones y alternativas. Bio-grafía, escritos sobre la biología y su enseñanza.13, 37-67.

Izquierdo, M., Espinet, M., García, M.P., Pujol, R. M. y Sanmartí, N. (1999). Caracterización y fundamentación de la ciencia escolar. Enseñanza de las ciencias. Número extra p.79-89.

Lopez, A., y Moreno, G. (2014). Sustentación teórica y descripción metodológica del proceso de obtención de criterios de diseño y validación para secuencias didácticas basadas en modelos: El Caso del fenómeno de la fermentación. Biografía Escritos sobre la biología y su enseñanza, 7(13), 109-126.

Leach, J. \& Scott, P. (2002). Designing and evaluating science teaching sequences: An approach drawing upon the concept of learning demand and a social constructivist perspective on learning. Studies in Science Education. 38, 115-142.

Rodríguez, G. Gil, J. y García, E. (1996). Metodología de la investigación cualitativa. Archidona, España. Aljibe, S.L.

Nombre: Aura Yuseli Mena Gómez - Fanny Angulo Delgado

Correos: yurame1987@hotmail.com

auragomez1987@gmail.com

fanny.angulo@udea.edu.co 